\title{
Explicit F.E. formulation with modified linear tetrahedral elements applied to high speed forming processes
}

\author{
P.O. De Micheli ${ }^{1}$, K. Mocellin ${ }^{1}$ \\ ${ }^{1}$ CEMEF, UMR CNRS 7635 - 2 rue Claude Daunesse, 06904 Sophia-Antipolis CEDEX, France \\ URL: www.cemef.ensmp.fr
}

e-mails: Pascal.De_Micheli@ensmp.fr ; Katia.Mocellin@ensmp.fr

\begin{abstract}
A recent explicit FE formulation with modified linear tetrahedral elements is adapted for high speed metal forming simulation. This formulation both enables the use of efficient adaptive non structured meshers, and tackles the locking effect in quasi-incompressible cases. We implement this formulation for the infinitesimal elastic plastic case. The anti-locking modification effect will be underlined on two 3D bench marks: an elastic compression test and an elastic-plastic bar impact test.
\end{abstract}

Key words: explicit, anti volumetric locking, linear tetrahedrons, plasticity, high-speed metal forming

\section{INTRODUCTION}

Explicit codes using simple hexahedral elements have exhibited a great success in impact and metal forming simulations in the last decades. They allow dealing easily with complex material behaviours and contact cases. Unfortunately, automatic remeshing algorithms on hexahedra are not as efficient as on tetrahedra, and may fail for complex geometries. In addition, the standard linear tetrahedral element exhibits volumetric-locking effect for incompressible and quasi-incompressible materials simulations (such as large plastic deformations for example).

Several authors have proposed different methods in order to tackle the volumetric locking problem on tetrahedral elements.

Mixed elements (such as the mini-element $\mathrm{P} 1{ }^{+} \mathrm{P} 1$ ) or second order elements would avoid locking effect, but the numerical cost would be too big for explicit simulations.

B-bar or F-bar methods [1] need the definition of element patches, and are therefore not so automatic.

Characteristic-Base Split (CBS) methods, imported from fluid dynamics [2,3] is a good approach to avoid locking with time inexpensive methods. It is yet quite widely used.

The idea of pressure average in finite elastic-plastic strain is presented in reference [4]. This formulation seems to have a bigger critical time step than the CBS formulation and similar results [5]. This formulation is modified in [6], by working on the spherical part of the strain instead of the pressure. This more general formulation facilitates the dealing of multi-material simulations, is only introduced for infinitesimal elasticity without remeshing.

Recently, strain averaged formulations are proposed to avoid both volumetric and bending locking $[7,8]$. But those formulations need to be stabilized for hourglassing, and no solution is proposed for explicit formulations for the moment [9].

This article introduces the extension of the explicit infinitesimal elastic formulation introduced in [6], to infinitesimal elastic-plastic case with remeshing possibilities.

The modified anti-volumetric-locking linear tetrahedral element will be first described (paragraph 2 ). Then, the anti-locking effect of this formulation will be underlined on elastic (paragraph 3) and elastic-plastic (paragraph 4) 3D benchmarks. Finally, we will rapidly conclude on current abilities of the formulation, possible enhancements and on its interests for high-speed metal forming simulations. 
2 FORMULATION OF THE MODIFIED ANTIVOLUMETRIC-LOCKING LINEAR TETRAHEDRON

\subsection{Notations}

Table1. Notations used in this article

\begin{tabular}{|c|c|}
\hline Variable & Signification \\
\hline$v$ & nodal velocity \\
\hline$x$ & time step \\
\hline$\Delta t$ & element volume \\
\hline$J$ & $\begin{array}{c}\text { ratio of total element volume variation } \\
\text { averaged on the adjacent elements }\end{array}$ \\
\hline $\mathcal{E}, \widetilde{\mathcal{E}}$ & $\begin{array}{c}\text { non modified and modified infinitesimal } \\
\text { strain tensor }\end{array}$ \\
\hline$\dot{\mathcal{\varepsilon}}$ & strain rate tensor \\
\hline $\begin{array}{c}\text { exponent "node" } \\
\text { or "elt" }\end{array}$ & precise if the value is nodal or elemental \\
\hline subscript & precise the time step number of the value \\
\hline$f^{\text {ext }}, f^{\text {int }}$ & external and internal nodal force vectors \\
\hline
\end{tabular}

\subsection{General presentation of the explicit formulation}

Our formulation is based on a classical explicit resolution of the dynamical mechanical equations, with a lumped mass matrix. We use a first order explicit central differences time integration scheme (equations 2, 3). Our plastic solver uses an isotropic radial return map algorithm. Classical Rayleigh damping type and linear bulk viscosity are used to damp at least high frequency oscillations.

\subsection{The anti-volumetric-locking formulation}

This formulation is based on a standard linear tetrahedral explicit formulation, with a modification of the infinitesimal strain tensor by substituting its spherical part by the ratio of total element volume variation averaged on the adjacent elements, $J$.

$\widetilde{\mathcal{E}}=\mathcal{\varepsilon}-\left(\frac{1}{3} \operatorname{tr} \mathcal{\varepsilon}\right) \cdot I d+\left(\frac{1}{3}(J-1)\right) \cdot I d$

Considering standard linear tetrahedra, the volume variation constraint is imposed at each element. In a classical 3D mesh, there are about 6 times more elements than nodes. Thus, in case of quasiincompressibility, the problem is over-constrained and locking occurs. In our modified formulation, the volume variation constraint is only imposed at each node. It is enough to decrease sufficiently the number of constraints to avoid locking in cases of quasi-incompressibility.
We use a single method in order to both update the ratio of total volume variation of the elements and to average it on the surrounding elements. This method allows updating the averaged volume variation $J$ without cumulating errors dues to the update procedure.

At each time step, the increment ratio of volume variation is computed for each element. Then, it is interpolated at the nodes of the mesh. Next, it is possible to deduce the new ratio of the total variation of volume using the nodal value at the precedent time step (that could have been remapped in case of remeshing) and multiplying it by the current increment ratio of volume variation. Finally, the updated element averaged ratio of total volume variation is interpolated at the integration point of each element using the nodal values and the shape functions. The mathematical description of this procedure can be seen in the next section, equations (6) to (8).

\subsection{Programming box:}

The steps to solve a time increment from time step $n$ to time step $n+1$ are summarized here:

a) Explicit nodal resolution and coordinates update :

$$
\begin{aligned}
v_{n+1 / 2} & =v_{n-1 / 2} \\
& +\Delta t\left\{\sum_{\text {node } \in \text { elt }} \frac{\rho \cdot V_{n}^{e l t}}{\operatorname{dim}+1}\right\}^{-1}\left(f_{n}^{e x t}-f_{n}^{\mathrm{int}}\right) \\
x_{n+1}= & x_{n}+\Delta t \cdot v_{n+1 / 2}
\end{aligned}
$$

b) Deduction of strain rate and infinitesimal strain tensors:

$$
\begin{aligned}
& \dot{\varepsilon}_{n+1 / 2}=\operatorname{sym}\left(\operatorname{grad}\left(v_{n+1 / 2}\right)\right) \\
& \varepsilon_{n+1}=\varepsilon_{n}+\dot{\varepsilon}_{n+1 / 2} \cdot \Delta t
\end{aligned}
$$

c) Calculation of the nodal incremental volume variation using a non weighted average:

$\left[\frac{V_{n+1}}{V_{n}}\right]^{\text {node } i}=\sum_{\text {elt }}^{i \in \text { elt }} \frac{1}{\operatorname{dim}+1}\left[\frac{V_{n+1}}{V_{n}}\right]^{\text {elt }}$

d) Updating of the ratio of the nodal total volume variation: 


$$
\left[\frac{V_{n+1}}{V_{0}}\right]^{\text {node } i}=\left[\frac{V_{n+1}}{V_{n}}\right]^{\text {node } i} \times\left[\frac{V_{n}}{V_{0}}\right]^{\text {node } i}
$$

e) Deduction of the averaged element total volume variation $\mathrm{J}$ :

$$
\left[J_{n+1}\right]^{\text {elt }}=\sum_{\text {node }}^{i \in \text { elt }}\left(\frac{1}{\operatorname{dim}+1}\left[\frac{V_{n+1}}{V_{0}}\right]^{\text {node } i}\right)
$$

f) Modification of element strain:

$$
\widetilde{\mathcal{E}}_{n+1}=\varepsilon_{n+1}+\frac{1}{3}\left\{-\operatorname{tr} \varepsilon_{n+1}+\left(J_{n+1}-1\right)\right\} \cdot I d
$$

g) Classical mechanical calculus of element stress from modified strain tensor:

$$
\sigma_{n+1}=f\left(\widetilde{\varepsilon}_{n+1}, \text { internal variables }\right)
$$

\section{ELASTIC COMPRESSION TEST}

\subsection{Description of the test}

The test consists in the $20 \%$ longitudinal compression of a cylinder. The material is linear elastic and quasi-incompressible (Young modulus: $3000 \mathrm{~Pa}$; Poisson ratio: 0.49). The initial dimensions of the cylinder are $10 \mathrm{~mm}$ diameter and $10 \mathrm{~mm}$ height. The results are observed when the equilibrium state is reached. The contact is nonsliding. In this case, volumetric locking is likely to appear in the region near the tools [6].

\subsection{Results}

We can now analyse the effect of anti-locking modification. First, the stress is stabilized and the over-estimation of the stress in the region near the tools is tackled. We can observe for example the longitudinal stress repartition in the parts obtained with standard linear tetrahedra and modified linear tetrahedra in figure 1. In the standard formulation, a checkerboard effect is visible in region near the tools. This problem is solved in the modified formulation. The repartition of longitudinal stress in the cut is also much smoother for the modified formulation than for the standard one. We should also notice than we use the same scale for figures 1.A and 1.B. This scale is adapted to figure 1.B. However, in 1.A, the range of values is 4 times greater (the minimum value is $868 \mathrm{daPa}$ and the maximum value is $+398 \mathrm{daPa}$ ).

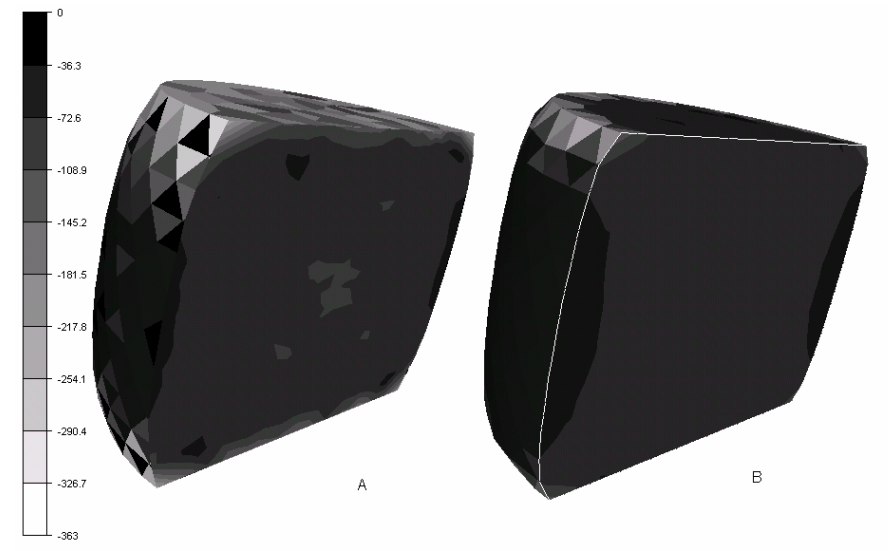

Fig. 1. Longitudinal stress in daPa in the elastic part for $20 \%$ longitudinal compression after that the equilibrium is reached, for standard linear tetrahedra (A) and modified linear tetrahedra (B).

Then, geometrical effects of locking are tackled as well. Final radii of the cylinder versus the height are plotted in figure 2, for both modified and unmodified formulations. The non-modified linear tetrahedron underestimates the deformation of the elements in contact with the tools. Those elements lock. This problem is solved when using the modified formulation. We obtain, for both modified and non-modified formulations the same final shapes as in reference [6].

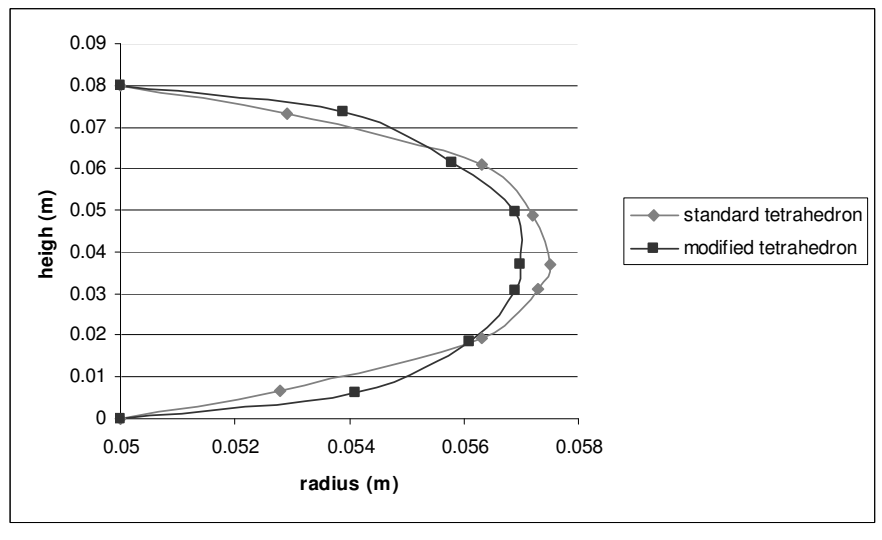

Fig. 2. Comparison of the final cylinder shapes (radius along the height) for the standard linear tetrahedron and the modified linear tetrahedron.

\section{ELASTIC-PLASTIC BAR IMPACT TEST}

\subsection{Description of the test}

This elastic-plastic benchmark is very often studied to test explicit codes and anti-volumetric-locking formulations, because of its dynamics characteristics and its high sensibility to volumetric locking $[2,4,5,9]$. A cylindrical copper rod $(32.4 \mathrm{~mm}$ length and $3.2 \mathrm{~mm}$ radius) moving at $227 \mathrm{~m} / \mathrm{s}$ impacts a 
rigid wall. There is no friction between the wall and the rod. The copper is supposed to be elastic-plastic with linear hardening (Young modulus: $117 \mathrm{GPa}$; Poisson ratio: 0.35; initial yield stress: $0.4 \mathrm{GPa}$; hardening modulus: $0.1 \mathrm{GPa}$ ).

\subsection{Results}

In the bar impact test, geometrical effects of the antilocking modification are easily visible (see cuts in figure 3 ). With standard tetrahedra, the strain is not localized at the impact location as it should be, but is scattered in the whole rod. The final impact radii of cylinders are $5.85 \mathrm{~mm}$ for standard linear tetrahedra and $7.09 \mathrm{~mm}$ for modified linear tetrahedra. Those results are in good agreement with impact radii obtained in the literature (between 7.0 and $7.1 \mathrm{~mm}$ ) $[2,4,5,9]$.

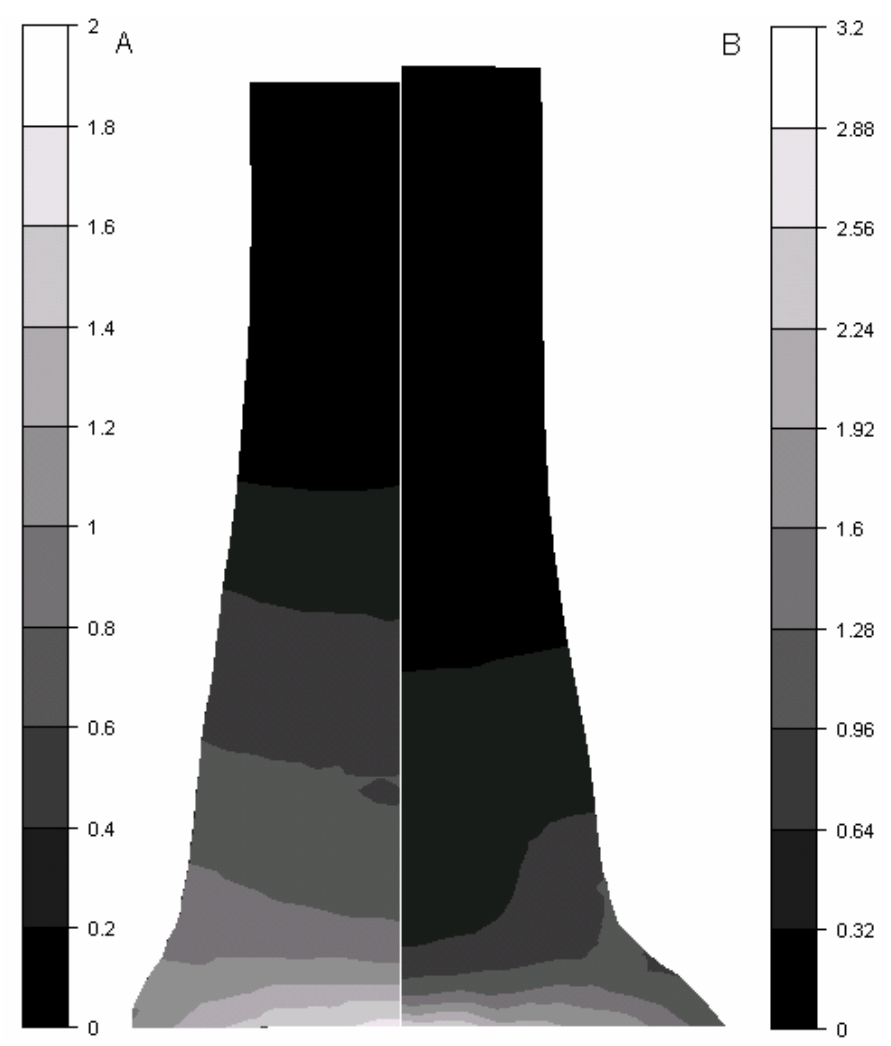

Fig. 3. Equivalent plastic strain in an axial cut of the rod at the end of the bar impact test for standard linear tetrahedra (A) and modified linear tetrahedra (B).

\section{CONCLUSIONS}

In this article, we extend an anti-volumetric-locking modification proposed in reference [6], inspired from "averaged nodal pressure" formulations [4], to infinitesimal plasticity. A very simple and thus quick formulation is obtained, that can be used with automatic adaptive remeshing. The hypothesis of small deformations is justified even for quite important final strain, because of the small critical time step in explicit metal forming simulations.

However, great rotations are not taken in account, and the formulation can't deal with bending locking. An "averaged nodal strain" formulation could be envisaged, but no explicit anti-hourglassing stabilization is proposed for it yet $[7,8,9]$. Some critical cases must then be considered with care.

This promising formulation will be now coupled with contact and thermal analysis, and tested on various complex high speed metal forming processes.

\section{ACKNOWLEDGEMENTS}

The authors would like to thanks the French PACA region, Transvalor ${ }^{\odot}$ and the French Industrial and Mechanical Technical Centre for financial support.

\section{REFERENCES}

1. E.A. de Souza Neto, F.M. Andrale Pires and D.R.J. Owen, F-bar-based linear triangles and tetrahedra for finite strain analysis of nearly incompressible solids. Part I: formulation and benchmarking, Int. J. Numer. Meth. Engng, 62, (2005) 353-383

2. O.C. Zienkiewicz, J. Rojek, R.L. Taylor and M. Pastor, Triangles and tetrahedral in explicit dynamic codes for solid, Int. J. Nummer. Meth. Engng, 43, (1998) 565-583

3. J. Rojek, E. Onate and R.L. Taylor, CBS-based stabilization in explicit solid dynamic, Int. J. Numer. Meth. Engng, 66, (2006) 1547-1568

4. J. Bonet and A.J. Burton, A simple averaged nodal pressure tetrahedral element for incompressible and nearly incompressible dynamic explicit applications, Commun. Numer. Meth. Engng, 14, (1998) 437-449

5. J. Bonet, H. Marriott and O. Hassan, Stability and comparison of different linear tetrahedral formulations for nearly incompressible explicit dynamic applications, Int. J. Numer. Meth. Engng, 50, (2001) 119-133

6. G.R. Joldes, A. Wittek and K. Miller, Improved Linear Tetrahedral elements for Surgical Simulations, In: Proc. MICCAI 2006, 1, (2006), 54-65

7. C.R. Dohrmann, M.W. Heinstein, J. Jung, S.W. Key and W.R. Witkowski, Node-based uniform strain elements for three-node triangular and four-node tetrahedral meshes, Int. J. Numer. Meth. Engng, 47, (2000) 15491568

8. J. Bonet H. Marriott and O. Hassan, An averaged nodal deformation gradient linear tetrahedral element for large strain explicit dynamic applications, Commun. Numer. Meth. Engng, 17, (2001) 551-561

9. M.A. Puso and J. Solberg, A stabilized nodally integrated tetrahedral, Int. J. Numer. Meth. Engng, 67, (2006) 841-867 